\title{
João Guimarães Rosa et la France
}

\section{La réception toujours recommencée}

Si João Guimarães Rosa a d'abord été (discrètement) reconnu comme poète, avec un recueil qui reçut en 1936 le prix de l'Académie Brésilienne des Lettres ${ }^{1}$, c'est comme prosateur qu'il s'est imposé au Brésil. Dix ans après les poèmes de Magma, les neuf nouvelles de Sagarana remportèrent un solide succès, qui ne fit que s'amplifier en 1956, quand parurent coup sur coup le vaste ensemble Corpo de baile et son roman Grande sertão : veredas. Le doute n'était plus permis : un grand auteur, une œuvre déjà ample et puissante ... Entre temps, il y avait eu le concours Humberto de Campos organisé en 1937 par la maison d'éditions José Olympio où, sous le pseudonyme de Viator, il avait décroché, à une voix près, une seconde place derrière Luís Jardim. Le juré Graciliano Ramos s'en expliqua : "Trois membres de la commission [dont lui-même] ont choisi un livre qui ne croît ni ne décline » (Guimarães Rosa 1994 : 110). Le contraire des nouvelles concurrentes jugées inégales, mais dont quelques sommets enthousiasmèrent le sagace Prudente de Morais, comme Conversa de bois, une conversation entre bovins qui migrera dans Sagarana. Il fallut donc un temps pour que Guimarães Rosa devienne prophète en son pays, et que soit comprise, assimilée son audacieuse nouveauté, qui inventait une troisième voie entre la tradition régionaliste et un style quasi expérimental, recréé de toutes pièces, une langue concrète, enracinée, et des soubassements savants et métaphysiques. Le tout autour d'une réalité aussi tangible et géographique qu'abstraite et insaisissable, le sertão.

Rien de plus brésilien, mais aussi rien de moins universel. Dès lors, une fois la reconnaissance nationale établie, une question se posait : comment cette œuvre allait-elle traverser les frontières ? Sur le continent américain, comme en Europe, l'intérêt des éditeurs commença à frémir dès la fin des années cinquante. Mais il

1 À vrai dire, en 1929, il reçoit un premier prix (ex aequo avec l'historien et critique Nelson Werneck Sodré) pour trois nouvelles, lors d'un concours organisé par le magazine $O$ Cruzeiro. Il a alors 21 ans.

Márcia Aguiar, Unifesp

Michel Riaudel, Sorbonne Université

Ә Open Access. (C 2022 Márcia Aguiar and Michel Riaudel, published by De Gruyter. (c))BY-NC-ND This work is licensed under the Creative Commons Attribution-NonCommercial-NoDerivatives 4.0 International License. https://doi.org/10.1515/9783110707557-010 
restait bien des obstacles à surmonter, et une série d'embûches que cet article va s'employer à retracer pour ce qui sera de sa destinée française ${ }^{2}$.

\section{Au seuil des années 1960}

La France se reconstruit, le monde se restructure. On a parlé, pour cette période, des Trente Glorieuses, pour désigner l'effervescence économique qui a succédé au chaos et à la désolation issus du conflit mondial des années quarante. L'Europe est et n'est plus, ne peut plus être, le centre : des modèles ont failli, les lignes de fracture se déplacent, autour de deux axes. Est/Ouest. C'est l'espérance d'un monde plus juste contre une certaine idée de la modernité, l'humanisme contre la technè, le collectif contre l'individu ..., tensions diverses que deux blocs incarnent à leur manière, sortis de la guerre chaude pour basculer dans la guerre froide. À cette topographie se superpose un axe Nord/Sud, même s'il ne porte pas encore ce nom : celui de la décolonisation, concédée ou conquise par les armes. Les marges entrent en scène. Une soif d'auteurs étrangers s'empare des maisons d'édition, en quête de nouvelles voix. Calmann-Lévy crée sa collection « Traduit de », Le Seuil ses « Pierres vives ». Avant de se spécialiser dans les guides de voyage, l'éditeur suisse Nagel publie Jorge Amado et d'autres dans sa ligne des « Grands romans étrangers ». Le résistant Pierre Seghers donne à lire les « Poètes d'aujourd'hui », dont les Brésiliens Vinícius de Morais, Ribeiro Couto, Manuel Bandeira, Murilo Mendes. Des écrivains dirigent de nouveaux domaines : Gallimard confie les « Littératures soviétiques » à Louis Aragon et les littératures latino-américaines, placées sous le signe de « La Croix du Sud », à Roger Caillois, tandis que Marcel Duhamel explore le roman noir étasunien ... Le nombre de traductions double plus ou moins chaque année de 1944 à 1948, en passant de 81 titres à 1088, ce qui devient l'étiage annuel moyen pour les années cinquante (Mollier 1995 : 373-394).

Dans ce nouvel horizon, l'Amérique latine et les Caraỉbes occupent une place de choix. Tiraillées entre la « protection du grand frère », au nord, et des envies de socialisme, nourries par les substrats amérindiens ou noirs mais façonnées par l'ancienne colonisation européenne, en particulier ibérique, engagées dans le « développement » mais encore profondément rurales, elles offrent

2 Deux fonds ont été particulièrement explorés par Márcia Aguiar : les archives de l'Institut d'études brésiliennes de l'Université de São Paulo (IEB-USP), référencées désormais comme « Fonds JGR - IEB-USP », et celles de l'Institut Mémoire de l’Édition Contemporaine : Fonds Seuil - IMEC. 
un terrain rêvé à la France et l'Europe du sud, pour des utopies compensatrices faisant contrepoids à la domination yankee, protestante, anglo-saxonne, tout comme à son urbanisation galopante. Tiers-mondisme et non alignement, Cuba et Tricontinentale vont structurer une partie du paysage culturel et intellectuel. C'est dans ce contexte, prêtant à toutes les attentions et quelques malentendus, que surgit sur la scène internationale, et en particulier française, l'œuvre de João Guimarães Rosa. Dès 1958, sa dernière histoire de Sagarana, " A hora e vez de Augusto Matraga », est traduite en espagnol et en français (Aguiar/Riaudel 2018) ${ }^{3}$.

"L'heure et la chance d'Augusto Matraga » intègre l'anthologie Les vingt meilleures nouvelles de l'Amérique latine, éditée par Pierre Seghers. L'éditeur s'était signalé pendant la guerre avec ses livraisons des Poètes casqués, puis en 1944 par le premier volume des « Poètes d'aujourd'hui » consacré à Paul Éluard. Un format poche, grand public, une fenêtre sur l'étranger, que va prolonger la collection «Autour du monde ». Le volume qui accueille le premier texte de Guimarães Rosa en France a d'abord été conçu par l'écrivain vénézuélien Juan Liscano comme une sélection de «Vingt nouvelles choisies de l'Amérique latine ». Il est précisé avant même la page de faux-titre : "Pour entrer dans le cadre de la collection Melior, l'éditeur a modifié le titre ».

Juan Liscano explique avoir limité son choix « à la génération d'avantgarde du lendemain de la première guerre mondiale, et à quelques écrivains qui, bien que plus jeunes, peuvent être intégrés dans cette tendance » (Liscano 1958 : 16). Du Brésil, il retient Mário de Andrade et Guimarães Rosa, que traduisent Antonio Tavares Bastos (lié à l'Unesco) et sa femme Georgette. La notice qui précède la nouvelle met en garde contre la tentation d'une réduction régionaliste et préfère rapprocher l'auteur de la chanson de geste « adapté[e] évidemment à la réalité sociale et au milieu, de Minas Gerais ». Sa « langue malléable, sonore, poétique, d'inspiration tantôt populaire, tantôt érudite », [...] répond au climat fabuleux dans lequel agissent ses hommes d'une mythologie de racine nettement humaine et américaine. » On cite aussi anonymement un rapprochement avec Joyce, mais « un Joyce très brésilien ». Une partie du cadre de cette première réception est déjà posée. Le lecteur français se ré-enchantera au merveilleux sans perdre de vue le terrain social.

3 Pour une analyse de la réception de Guimarães Rosa au plan international, voir Aguiar, Márcia/ Riaudel, Michel (2018) « João Guimarães Rosa : un auteur à remémorer pour le commémorer ». In Herrera, Raquel Molina/Castaño, Victoria Ríos (eds.) Colloquia. Paris : Sorbonne Université. Disponible en ligne : https://colloquiasal.files.wordpress.com/2019/03/colloquia6f.pdf et Aguiar, Márcia V. M. de (2017): « A publicação de Guimarães Rosa na França dos anos 1960 ». In Reimão, Sandra/ Riaudel, Michel (eds.), Livros, literatura e história: passagens Brasil-França. Florianópolis: Escritório do Livro, pp. 79-102. 
Il n'est pas exclu que Pierre Seghers lui-même ait déjà eu alors connaissance du nom de Guimarães Rosa à travers Vinícius de Moraes. Le poète et diplomate (comme l'auteur de Sagarana), que Seghers avait édité en 1953, l'avait invité à visiter le Brésil en juillet 1957 . L'éditeur français confie à Antonio Tavares Bastos qu'il considère cette histoire d'Augusto Matraga comme " une nouvelle absolument extraordinaire ». Le 6 mai 1958, il écrit à l'auteur pour lui vanter son « texte singulièrement attachant » (Lettre à João Guimarães Rosa 6 mai 1958) et prendre une option sur les droits de Grande sertão : veredas destiné à trouver sa place dans la collection des "Grands romans ». Trop tard. Albin Michel était passé avant et avait obtenu l'accord de Guimarães Rosa dès le mois de mars ${ }^{5}$.

L'écrivain brésilien adresse néanmoins à Seghers un exemplaire de Sagarana en lui proposant de traduire le recueil (Lettre à Pierre Seghers 5 juillet 1958). Mentionnant ses « conversations avec des amis brésiliens du monde littéraire », Seghers insiste :

Mieux que Sagarana, qui est un recueil de contes, il semble que Grande sertão : veredas réponde exactement à la ligne de ma maison d'éditions : une grande œuvre romanesque où le travail sur le langage, l'invention, et une recréation du monde à travers les mots, le dialogue du bien et du mal constituent une présence unique, la vôtre (Lettre à João Guimarães Rosa 5 jul. 1958).

Les négociations avec Albin Michel aboutissent début 1959, et Guimarães Rosa en informe Pierre Seghers et fait part de ses regrets, tout en le remerciant d'avoir été le premier éditeur à diffuser son œuvre en Europe :

Je le regrette profondément, non seulement à cause de cela, mais aussi parce que vous, qui êtes un grand compagnon des lettres et sincère ami du Brésil et des écrivains brésiliens, vous avez été le premier à présenter mon nom et mon œuvre en France, dans votre belle anthologie (Lettre à Pierre Seghers 15 fév. 1959).

Seghers n'est pas le seul frustré de ce dénouement. En cette même année 1958, les Éditions du Seuil ont entrepris des démarches pour publier le roman tant convoité. La maison dirigée par Paul Flamand et Jean Bardet, qui est en plein essor, s'est donné pour mission de « trouver des auteurs neufs pour des problèmes présents " (Flamand $1979:$ 7). La collection « Pierres Vives », créée dès 1945, témoigne de cette ouverture : Maurice Nadeau, Pierre Klossowski, Roland Barthes, y font leurs débuts (Serry 2008 : 32). Plus tard de nouvelles collections

4 Cf. Correio da manhã, 27 juillet 1957 (Hemeroteca Nacional) et la lettre de Pierre Seghers à Antonio Tavares Bastos, 25 juin 1957 (Fonds JGR - IEB-USP).

5 Lettre des éditions Albin Michel à José Olympio Editores, 23 mars 1958. Fonds JGR - IEB-USP. 
creuseront ce sillon : «Tel Quel » (1963), « L'ordre philosophique » (1964), « Le champ freudien » (1964), « Poétiques » (1970). Le catalogue est à l'aune de cette volonté de participer activement au débat d'idées contemporain et à la formation d'un nouvel homme. La littérature étrangère y joue un rôle clé. En 1951, le succès du roman de Giovanni Guareschi, Le Petit Monde de Don Camillo, assure à la maison les bases financières pour des paris plus risqués en attirant de plus ou moins jeunes auteurs du monde entier (Serry 2008 : 42). Peter Weiss, Heinrich Böll, Günther Grass, Robert Musil pour le domaine germanique ; Italo Svevo, Giuseppe Lampedusa, Italo Calvino, Umberto Eco, côté italien. Pour le domaine anglosaxon, Monique Nathan fait découvrir John Updike. Le domaine francophone n'est pas oublié du « Cadre rouge » : Édouard Glissant, André Schwarz-Bart, Léopold Sédar Senghor («Pierres vives ») ; dans la collection « Méditerranée », Emmanuel Roblès, Kateb Yacine, Mouloud Feraoun, Mohammed Dib (Mollier 2012). Écrivains européens et du tiers-monde sont sur un pied d'égalité. À partir de 1958, le « Cadre vert ", une couverture dessinée par Pierre Faucheux, distingue les auteurs étrangers contemporains non francophones, comme pour effacer les aires linguistiques et constituer un espace littéraire sans frontières (Serry 2002 : 75). La collection intègre ainsi d'autres ensembles de la maison d'édition, sans rien sacrifier de l'exigence de qualité qui caractérisait déjà les traductions du « Don des Langues ». Contraintes de renoncer au roman de Guimarães Rosa, les éditions du Seuil vont jouer néanmoins un rôle fondamental dans la diffusion de son œuvre en livrant au public français la série de Corpo de baile. Le liseré vert encadre Buriti, le premier des trois sous-ensembles parus dans les années soixante, et qui réunit « La fête à Manuelzão », « Dão-lalalão », et « Le message du morne » : selon l'écrivain brésilien, une « traduction magistrale », pour son premier ouvrage " livré au monde grand » (Lettre à Jean-Jacques Villard 27 jul. 1961), dont les droits ont commencé à être négociés dès la fin de l'année 1957.

Le jeune éditeur Michel Chodkiewicz avait écrit alors à José Olympio Editores pour signifier son intérêt de publier des auteurs brésiliens en France. La maison carioca transmet l'adresse de Guimarães Rosa, puisque l'achat des droits doit se faire directement avec l'écrivain. À la lettre de Chodkiewicz du 3 mars 1958, Guimarães Rosa suggère, faute de pouvoir offrir son roman, que le Seuil examine Corpo de baile, sept longues nouvelles « de la brousse brésilienne » : " J'ai encore disponibles les droits français d'un autre livre, “Corpo de Baile” (sept nouvelles, en deux volumes, 822 pages), paru aussi en 1956, ce dont, le cas échéant, je pourrais vous accorder l'option » (Lettre à Michel Chodkiewicz 5 jul. 1958). Après quelque vaine insistance pour rattraper le roman, Chodkiewicz accepte (en postscriptum, comme un remords résigné) d'étudier les nouvelles : « Pouvez-vous m'envoyer également le roman que vous avez publié en 1956, et dont vous me 
parlez dans votre dernière lettre (Lettre à João Guimarães Rosa 17 oct. 1958). Guimarães Rosa lui expédie un exemplaire le 10 novembre 1958 :

Pour ce qui est de mon ouvrage Corpo de Baile, je suis en train de vous le faire parvenir, par courrier maritime, en pli séparé, recommandé. Comme je vous avais déjà dit, ce n'est pas un roman, mais il s'agit d'une série de grandes nouvelles. Je me permets cependant de vous dire que, ici, plusieurs personnes l'ont considéré du même niveau que Grande Sertão : Veredas, et il y en a même ceux [sic] qui le préfèrent de beaucoup à celui-ci (Lettre à Michel Chodkiewicz 10 nov. 1958).

Les avis des lecteurs du Seuil sur Corpo de baile sont enthousiastes. Et Chodkiewicz écrit à leur auteur le 27 janvier 1959 :

Je pense pouvoir vous faire connaître, d'ici une semaine ou deux, au plus, notre décision définitive. Mais, dès à présent, je tiens à vous informer du très vif intérêt que nous avons pris à la lecture de Corpo de Baile. Tous les lecteurs que j'ai sollicités sont unanimement favorables et considèrent votre roman comme une des plus grandes, voire même la plus grande des œuvres brésiliennes contemporaines (Lettre à à João Guimarães Rosa 27 jan. 1959).

La décision définitive devait venir du Comité de Lecture du Seuil, composé de son directeur littéraire, Paul Flamand, et des autres éditeurs de la maison : Jean Cayrol, Paul-André Lesort, Claude Durand, Philippe Sollers, Luc Estang, Chris Marker et Michel Chodkiewicz. Le 13 février 1959, Guimarães Rosa reçoit un télégramme. Le Seuil souhaite non seulement faire paraitre Corpo de baile mais devenir son éditeur exclusif en France, s'engageant à publier tous ses ouvrages, y compris ceux à venir :

SOUHAITONS DEVENIR VOTRE EDITEUR UNIQUE EN FRANCE. DESIRONS PUBLIER CORPO DE BAILE STOP GRANDE SERTAO STOP ET EUVRES SUIVANTES. VOUS ASSURONS PROFONDE ADMIRATION ET TOTAL DEVOUMENT CHODKIEWICZ EDITIONS DU SEUIL (Télégramme à João Guimarães Rosa 13 fév. 1959).

Cela correspond à une politique de la maison que Paul Flamand explicite dans l'un des bulletins des éditions : " le choix d'un manuscrit n'est pas d'abord un acte de commerce, ou bien un coup de dés ; c'est d'abord un engagement personnel, l'acceptation d'une aventure commune ». Avec son éditeur, le romancier « n'est plus seul face à la critique, face au public [...] il y a auprès de lui quelqu'un qui l'a choisi et dont la fidélité ne cesse pas (Lacouture 2010 : 93-94). Chodkiewicz ne dit pas autre chose à Guimarães Rosa, quand il affirme : " Nous voudrions être votre éditeur, et non pas un éditeur parmi d'autres ».

Vous savez, maintenant, que nous sommes fermement décidés à publier Corpo de baile. Comprenez qu'il ne s'agit pas, pour nous, d'une décision banale, mais que nous avons le désir de nous engager à fond sur votre nom et sur votre œuvre. Nous sommes prêts à faire 
pour vos livres, en France, ce que nous avons déjà fait, avec succès, pour ceux de Robert Musil et de Eliot. Nous savons que nous nous trouvons en face d'une très grande œuvre de celles qu'un éditeur n'a pas souvent la chance de rencontrer au cours de sa carrière et nous sommes résolus à tenter tout ce qu'il sera possible de tenter pour l'imposer en France, et la faire bénéficier de l'audience qu'elle mérite (Lettre à João Guimarães Rosa 17 fév. 1959).

Les cas de T. S. Eliot et de Robert Musil avaient d'ailleurs dû surmonter un défi que l'œuvre de Guimarães Rosa impose elle aussi, celui de la traduction. Dans les deux premiers exemples, l'effort avait rapporté quelques lauriers : Pierre Leyris reçut à l'unanimité le prix Denyse Clairouin pour son Poésie d'Eliot. Et les longues années de travail de Philippe Jaccottet valurent à sa version de L'Homme sans qualités (plus de 2000 pages) le Prix du meilleur livre étranger (Serry 2002 : 74-75). Il restait à trouver la perle rare pour recréer en français la langue réinventée du sertão.

\section{Une œuvre en quête de traducteur}

Fort des rapports de lecture signalant la « qualité exceptionnelle » et la « très grande originalité » de Corpo de baile, Michel Chodkiewicz écrit à Roger Bastide, grand connaisseur de la littérature brésilienne ${ }^{6}$. Le sociologue le rassure à son tour sur la qualité de l'ouvrage, mais le met en garde : il va falloir trouver un traducteur à la hauteur des enjeux.

Je n’ai pas lu Corpo de Baile qui a paru après mon départ du Brésil. Mais j’ai lu le premier livre de João Guimarães Rosa, qui le classait parmi les plus grands écrivains du Brésil. Tous les Brésiliens que j'ai vus depuis sont d'accord pour le considérer comme actuellement le seul très grand. Mais je crois qu'il est très difficile de le traduire ; il utilise souvent les procédés de Joyce, c'est-à-dire, qu'il crée des mots nouveaux (j'ai lu une très intéressante étude sur ses règles de fabrication des vocables) et il faudrait trouver un traducteur qui serait capable de transposer cela en français... (Lettre à Michel Chodkiewicz 20 jan. 1959).

Trois mois après le début de cette mission difficile, Chodkiewicz commente la situation dans une lettre à Antonio Bandeira, l'un des lecteurs du Seuil :

Je ne vous cache pas que je suis, pour l'instant, sinon découragé du moins très embarrassé. Cette semaine encore, j'ai vu plusieurs écrivains brésiliens de passage à Paris, qui ont tous applaudi au choix que nous avons fait, mais se sont montrés très pessimistes quant aux possibilités d'adaptation. J'espère que vous serez plus réconfortant ! (Lettre à Antonio Bandeira 29 mai 1959).

6 Lettre de Michel Chodkiewicz à Roger Bastide, 16 janvier 1959. Fonds Seuil - IMEC. 
Quelques jours plus tard, il partage ses déboires avec Guimarães Rosa, dans sa quête encore inféconde $d u$ " héros intrépide » :

À propos de cette traduction, je dois vous dire que mes premières recherches, très actives pourtant, pour trouver un ou des traducteurs capables de rendre votre texte en français, ont été jusqu'ici infructueuses. Toutes les personnes que j'ai pressenties, et qui ont fait un bref essai (en s'entourant des conseils de Brésiliens compétents), ont finalement reculé devant les difficultés de votre livre. Je ne suis pas découragé pour autant, mais je crains fort que les délais de traduction ne soient, du fait de tous ces tâtonnements, allongés audelà de mes prévisions. (Lettre à Guimarães Rosa $1^{\mathrm{er}}$ juin 1959).

Les lettres que Chodkiewicz a envoyées à ces traducteurs pressentis et l'évaluation des essais éclairent les raisons du renoncement des uns et les insuffisances des autres. Les Brésiliens invoquent leur incapacité « à manier la langue française avec la même maîtrise que Guimarães Rosa manie la nôtre » (Alvim 1961, nous traduisons). Un Français souligne la nécessité de bien connaître le régionalisme brésilien, vu qu'il y a « dans Corpo de baile beaucoup trop d'expressions locales ${ }^{7}$ ». Le Seuil juge certaines traductions trop lourdes et " avec de véritables fautes de français ${ }^{8}$ "; d'autres adoptent « un ton trop littéral, trop scolaire » sans chercher à rendre en français la multiplicité de noms d'animaux qui peuplent la prose de Guimarães Rosa ${ }^{9}$; malgré un ton « fidèle et correct », certaines tentatives ne parviennent pas à insuffler chez le lecteur français « le grand vent de liberté et de poésie qui souffle à travers les pages de Guimarães Rosa $^{10} »$.

Enfin Villard vint. Au bout de près d'une année de recherches, Michel Chodkiewicz écrit en janvier 1960 à l'attaché culturel de l'Ambassade du Brésil à Paris, qu'il avait associé à sa prospection : satisfait, il a trouvé un traducteur qui lui paraît « répondre tout à fait aux exigences qu'impose l'adaptation d'une œuvre aussi complexe ». " Les pages que M. Villard a traduites, à titre d'essai, sont excellentes et je crois pouvoir espérer la publication au début de l'année prochaine d'un premier tome de Corpo de baile » (Lettre à Everaldo Dayrell de Lima 7 jan. 1960).

Jean-Jacques Villard s'était présenté au Seuil à la fin de l'année 1959. À 62 ans, il avait à son actif douze livres traduits de l'anglais, de l'allemand, du néerlandais

7 Lettre de Pierre Denis à Michel Chodkiewicz, 8 octobre 1959. Fonds Seuil - IMEC.

8 Lettre de Michel Chodkiewicz à Guimarães Rosa, $1^{\text {er }}$ juin 1959. Fonds Seuil - IMEC.

9 Évaluation de test de traduction du 18 août 1959. Fonds Seuil - IMEC.

10 Lettre de Michel Chodkiewicz à Olga Obry, 30 décembre 1959. Fonds Seuil - IMEC. Traductrice, Olga Obry est aussi l'autrice de Catherine du Brésil, filleule de Saint-Malo. Paris : Nouvelles éditions Latines, 1948. 
et du portugais, " ou plus exactement du brésilien ${ }^{11}$ ", précise-t-il. Sa carrière de traducteur avait commencé quatre ans auparavant avec la traduction du premier volume de la saga d'Érico Veríssimo, Le Temps et le Vent. Issu d'un milieu bourgeois prospère et cultivé, il doit sa connaissance du portugais à la branche maternelle de sa famille. Dans la première lettre à Guimarães Rosa, il écrit :

À demi brésilien par ma mère, Paulina Klingelhoefer, née à Tijuca, j’ai vécu à Paris, chez mes grands-parents, dans une atmosphère purement brésilienne, qui m'a permis d'apprendre la langue et de connaître les usages (Lettre à João Guimarães Rosa 7 juillet 1961).

La famille d'Adolpho Klingelhoefer, son grand-père, vice-consul du Brésil en France depuis 1899 et négociant de café, valorisait aussi bien les arts ${ }^{12}$ que les sports $^{13}$. Quant au grand-père paternel, Théodore Villard, c'était un ingénieur réputé qui partageait avec son beau-père, Alexandre Bixio, un goût pour la botanique $^{14}$. Dans son enfance, il apprend également l'allemand et l'anglais, une formation en langues qu'il perfectionne dans deux prestigieux lycées, les Lycées Carnot et Henri IV, comme il le confie à Guimarães Rosa :

Né à Paris, le 22.2.1897 de père français et de mère brésilienne, née à Tijuca. Apprends l'allemand et l'anglais dès l'enfance. J'entends toujours parler le brésilien chez ma grand-mère Klingelhoefer, née de Gomensoro, et vis chez elle au milieu de coutumes et d'usages brésiliens. J'étudie le latin, le grec et la philosophie au Lycée Carnot, puis au Lycée Henri IV. (Lettre à João Guimarães Rosa 12 nov. 1963).

Sa formation classique est interrompue par la Première Guerre mondiale : « La guerre de 1914. Je m’engage à 17 ans et demi le 07.09.1914, pars pour le front le 07.02.1915 comme “cabo” d'artillerie montée. Champagne, Argonne, Verdun. Années de grandes misères. La boue, les poux, le manque de sommeil, le froid,

11 Lettre de Jean-Jacques Villard à Michel Chodkiewicz, 25 novembre 1959. Fonds Seuil IMEC.

12 Cf. l'article « José Guilherme Klingelhoefer ». In Portugal, Dicionário histórico, https://www. arqnet.pt/dicionario/klingelhoefer.html.

13 Le frère de Paulina, Adolphe Klingelhoefer, est considéré le premier athlète brésilien à participer à des Jeux Olympiques. Cf. Pierre de Coubertin (1863-1937). Olimpismo, sélection de textes de Norbert Müller et de Nelson Schneider Todt, trad. Luiz Carlos Bombassaro, p. 770-771.

14 Cf. Théodore Villard (1900) : Les Fleurs à travers les âges et à la fin du XIX ${ }^{e}$ siècle. Paris : Armand Magnier. Médecin et naturaliste, Alexandre Bixio avait indirectement participé de la création de la Revue des Deux Mondes (Marie-Louise Pailleron (1919) : François Buloz et ses amis. La vie littéraire sous Louis Philippe. Paris : Calmann-Lévy, p. 92). Jean-Jacques Villard écrira aussi un livre sur son arrière grand-oncle : Nino Bixio, uomo, marinaio, patriota. La vita avventurosa dell'eroe garibaldino nel racconto del pronipote (Milan: Viennepierre Edizione, 2008). 
la dysenterie ». Il côtoie la mort et la folie, souvenirs qu'il émaille de quelques anecdotes livrées à son auteur. Ce goût du récit, perceptible aussi dans les petites histoires de paysans de son village normand, vont intéresser Guimarães Rosa :

Gostei, imensamente, e muito lhe agradeço, das duas « estórias » que me conta. Principalmente, a do pobre Roussi, e de sua megera de mulher. Está contada magnificamente. E é terrivel. Agora é que vejo como os contos de Maupassant eram reais e plausíveis, quando mostra a dureza de coração de certos camponeses. Impressionou-me, muito ${ }^{15}$ (Lettre à Jean-Jacques Villard 14 oct. 1963).

$\mathrm{Au}$ début de la Deuxième Guerre mondiale, Jean-Jacques Villard est capitaine à la section du chiffre du II Groupe d'Armées. Après la Libération, il officie au Service d'Information Américain et pour le gouvernement militaire tripartite en Allemagne $^{16}$. Lorsqu'il s'attellera à la traduction de Primeiras estórias - sans qu'elle ne soit publiée -, il se souviendra de son expérience du décryptage des messages :

Je suis parvenu à la moitié du livre et je dois avouer que je vous ai bien souvent traité mentalement de toutes sortes de noms peu courtois ! Enfin, après de nombreuses recherches méthodiques, dont la pratique de la cryptographie m'avait donné l'habitude, je me disais soudain : «Tiens, c'est un mot grec ! » ou encore : « C'est un mot de l'alchimie arabe ! » et j'éprouvais l'immense satisfaction de celui qui a résolu un problème ardu. (Lettre à João Guimarães Rosa 25 avr. 1967).

Il commence à traduire à sa retraite, un hobby tardif qui devient presque une profession, comme il l'écrit dans sa lettre du 12 novembre 1963 :

Maintenant, je suis en retraite. Mes trois enfants sont mariés, j’ai 8 petits-enfants. Ma grande occupation est de traduire. Cela avait commencé comme un « hobby » en 1956, c'est presque devenu une profession aujourd'hui. En 8 ans j'ai traduit 23 livres, sans compter diverses brochures, de l'anglais, de l'allemand, du hollandais, du portugais, j'ai lu et fait la critique de centaines de romans de ces langues, plus certains en espagnol.

De 1956 au début des années 1970, Jean-Jacques Villard aura en effet traduit plus d'une trentaine de livres, parmi lesquels ceux d'Autran Dourado, d'Aquilino Ribeiro, de Marco Denevi, d’Ernesto Sábato, de Juan Carlos Onetti, d’Ángel

15 « J'ai aimé, immensément, et je vous en remercie beaucoup, les deux histoires que vous me racontez. Surtout celle du pauvre Roussi, et de sa mégère de femme. C'est magnifiquement raconté. Et c'est terrible. Je vois maintenant combien les contes de Maupassant sont réels et plausibles, quand il montre la dureté de cœur de certains paysans. Cela m’a impressionné, beaucoup. » (Lettre à Jean-Jacques Villard 14 oct.1963).

16 Cf. Lettre de Jean-Jacques Villard à João Guimarães Rosa, 12 novembre 1963. Fonds JGR IEB-USP. 
María de Lera ${ }^{17}$, et du côté anglais et néerlandais, ceux J. R. Salamanca, Léon Uris et Simon Vestdijk ${ }^{18}$. Rien de commun, néanmoins, avec l'expérience de la traduction de Corpo de baile, de Grande sertão : veredas et de Primeiras estórias.

Cette activité le passionne, mais seule l'écriture de Guimarães Rosa est capable de le troubler et de l'absorber, le faisant vivre des réalités qui lui sont méconnues et lui inspirent une communion d'esprit entre auteur et traducteur :

Quand j’ai terminé une telle traduction je me sens désorienté, amputé en quelque sorte d'une partie de moi-même, de la meilleure peut-être, et je prends un certain temps pour retrouver mon équilibre. [...] Peut-être nos esprits sont-ils pour ainsi dire en communion et que c'est cela qui me permet de vous bien comprendre et d'aimer ce que vous aimez. Mon grand regret est de si peu connaître le Brésil, n'ayant fait qu'une brève escale à Rio en 1921, mais maintenant j'ai l'impression d'avoir vécu des années sur les rives du São Francisco, de l'Abaeté, dans la région de l'Urucuia, que j'ai suivi des boiades, bu le vin de buriti, et je ne puis vous exprimer ma reconnaissance pour m'avoir procuré ce sentiment (Lettre à João Guimarães Rosa 29 oct. 1962).

Il reste maintenant à Villard à communiquer son enthousiasme au lecteur, dont on ne peut apprécier les réactions sans le ponctuer de deux considérations. La première est que rares sont alors les Français à pouvoir apprécier Guimarães Rosa dans l'original. La lutte pour trouver un traducteur en témoigne, de même que le rapport de lecture confié par l'éditeur à un jeune écrivain et critique de langue espagnole, Javier Domingo. On imagine, à partir de là la faiblesse des relais existant en France, nullement comparables à la situation de la littérature en langue anglaise, allemande, espagnole ou italienne. Enseigné à l'université seulement depuis 1919, le portugais ne s'implantera que progressivement, et les concours de recrutement de l'enseignement secondaire (CAPES, agrégation) ne seront créés qu'au début des années soixante-dix ${ }^{19}$. Ces paramètres sont indispensables pour une compréhension large des processus de réception.

La deuxième remarque concerne la place de la littérature brésilienne, dont le premier essor de l’œuvre de Guimarães Rosa dans les années soixante mas-

17 Dourado, Autran (1967) : La Barque des hommes. Paris : Stock. Ribeiro, Aquilino (1971) : La Voie sinueuse, préface de João Gaspar Simões. Paris : Fondation Calouste Gulbenkian, Centre culturel portugais. Denevi, Marco (1968) : Rosaura vient à dix heures. Paris : Albin Michel. Sábato, Ernesto (1967) : Alejandra, préface de Witold Gombrowicz. Paris : Éditions du Seuil. Onetti, Juan Carlos (1970) : Trousse-vioques. Paris : Stock. De Lera, Ángel María (1969) : Les derniers Etendards. Paris : Albin Michel.

18 Salamanca, J. R. (1963) : Lilith. Paris : Stock. Uris, Léon (1960) : Trahison à Athènes. Paris : Seghers. Vestdijk, Simon (1963) : L'île au rhum. Paris : Gallimard.

19 Cf. Michel Riaudel : «Cent ans d'enseignement universitaire du portugais en France : une histoire à mettre en perspective ", à paraître dans un ouvrage sur les études aréales aux Sorbonne Université Presses, sous la direction de Miguel Rodriguez et David Marcilhacy. 
que en fait un certain reflux. Dans l'après-guerre, l'attention portée aux écrivains latino-américains profite autant aux hispanophones qu'aux lusophones. Or une dissociation s'opère précisément autour de 1960, quand le volume des traductions du portugais du Brésil ne cesse d'augmenter, mais avec un regard beaucoup social et politique que littéraire. Paradoxalement, donc, le boom dit latino-américain n’a guère bénéficié aux écrivains brésiliens. Guimarães Rosa est une rare exception, et il faudra attendre les années quatre-vingt pour assister à un « boom » de la littérature brésilienne (Riaudel 2017 : 11-47).

\section{L’accueil français des années 1960}

Dans son rapport de lecture de Corpo de baile pour Le Seuil, Javier Domingo affirme que « ce livre surprendra énormément en France et déroutera complètement la critique » (Domingo 1959), faute de point de comparaison. Deux ans plus tard, en 1961, à la sortie de Buriti, dont il signe la préface - son prénom est alors transcrit « Xavier »-, sa prédiction s'accomplit. Ce dépaysement a souvent plu, il a aussi parfois rebuté. Ainsi l'hermétisme et l'ésotérisme déconcerte Guy Dumur, dans France Observateur : "Alors qu'on s'attend à lire - et qu'effectivement on lit - de bons récits folkloriques, l'auteur cite Plotin et Ruysbroeck l'Admirable, tandis que le préfacier se lance dans des commentaires mystico-philosophiques un peu effrayants » (Dumur 1961). Témoin du tournant " sociologique » que nous venons de signaler, le critique attendait dans le " monde de chair et de rêves » de Guimarães Rosa la représentation de réalités sinon lyriques, du moins tangibles : «Sans vouloir qu'on tombe dans les simplifications brésiliennes des films de Marcel Camus mais en se souvenant de Machado de Assis et même de Jorge Amado, on souhaiterait connaître un Brésil peut-être moins lyrique, mais plus réel. » Les personnages sont « tout à fait apolitiques », ce qui empêche qu'on les " situe », c'est-à-dire qu'on les inscrive dans un temps et un espace précis. Tout cela manque en somme de pittoresque et de réalisme.

D’autres verront ce même verre à moitié plein. Dans Le Canard enchaîné, René Lefèvre note : " Le livre est une très agréable surprise. Car nous sommes sevrés d'écrivains sud-américains au point de croire que ce continent n'exporte que des guitaristes et des danseurs » (Lefèvre 1961). Beaucoup ont décidément en tête Orfeu negro, qui a reçu la Palme d'Or à Cannes en 1959. C'est aussi le cas de Roger Ténèze, dans La Voix du Nord : « João Guimarães Rosa représente le Brésil authentique et non pas sa façade à demi-africaine que le cinéma et une 
certaine musique ont rendu populaire. Nous sommes assez loin ici d'Orfeu negro, de ses noirs insouciants et mélomanes. » (Ténèze 1961).

Le roman naturaliste d'Aluísio Azevedo, Le Mulâtre, paru lui aussi en 1961, sert de point de comparaison à Marcel Brion. À ses yeux, l'influence du milieu, la question raciale, sont des discussions étrangères à Buriti qui, bien qu'enraciné dans le sertão, se concentre sur la nature métaphysique de l'homme :

Avec João Guimarães Rosa et Buriti nous abordons un Brésil tout à fait différent de celui d'Azevedo et de son mulâtre : un Brésil où les revendications sociales et les réformes n'ont plus d'importance, un Brésil actuel, et aussi beaucoup plus vieux, presque inchangé dans sa figure et dans son âme, plus ancien que la conquête espagnole ; on presque dire, d'avant l'apparition de l'homme tant ce sont les éléments qui vivent le plus intensément, tant la présence humaine paraît insolite et presque incongrue (Brion 1962).

Procédant lui aussi par comparaison avec d'autres auteurs brésiliens du XIX ${ }^{\mathrm{e}}$ siècle, en l'occurrence José de Alencar et Machado de Assis, le dramaturge et critique belge Georges Sion émet l'hypothèse que Buriti rassure ceux qui s'interrogeaient sur l'avenir de la littérature brésilienne. Dans Le Phare, de Bruxelles, il estime que ce livre, « tour à tour saisissant et bizarre, captivant et confus », bouscule nos habitudes au risque de déranger. Mais « on ne saurait nier la force mystérieuse, l'ampleur parfois cosmique de cette geste grandiose » (Sion 1961). Sa lecture est alors nettement induite par la préface de Xavier Domingo, dont l'influence mérite qu'on s'y attarde. On y relève quelques références clés, empruntées aux épigraphes de l'œuvre (Plotin, Ruysbroeck), ou ajoutées : Heidegger, la Genèse, saint François, Goya et surtout Virgile (que lui souffle un article d'Henriqueta Lisboa). Tout cela converge vers l'idée d'un réalisme panique (à l'opposé du " régionalisme » ou de " l'abus de la couleur locale », " piège qui guette toujours la littérature d'Amérique latine » (Domingo 1961 : 8), d'un dualisme immanent composé de la Nature (ou sertão) et de l'homme primitif, « Adam nu », sertanejo ... Cette œuvre renoue avec les vérités premières, archaïques, fréquente le mondes des « ombres » et se réconcilie avec un cosmos lavé des confusions et des perversions modernes. Et si dimension populaire il y a, c'est celle de l'innocence, ou de la poésie des troubadours. La régénérescence que nous offre l'Amérique latine, c'est donc encore une fois la tradition occidentale (Domingo parle explicitement de «tradition latine ») décapée du présent, de l'ancrage trivial, pour revitaliser des " totems », une « fruste liturgie », une écriture-gnose, une initiation magique.

Il faut s'arrêter sur l'image du retable qui constitue le fil conducteur de la préface, car elle noue le religieux, les apparences et la profondeur (dont le palmier buriti est l'interface, élément à la fois naturel et métaphysique), l'idée 
des fragments réunifiés dans le Tout (« le sertão, c’est le Tout », conclut Domingo), tout en servant habilement un métarécit de la genèse éditoriale. Seuil, inconsolable d'avoir vu le grand monument romanesque lui échapper et de devoir se contenter de nouvelles, semble de la sorte récrire l'histoire. Domingo $\mathrm{y}$ insiste :

Buriti, nous l'avons dit, est un vaste retable, divisé en six panneaux ${ }^{20}$. Techniquement à la manière des retables de Jérôme Bosch, par exemple - ce livre est un tout homogène dont pourtant chaque partie peut être proposée au lecteur indépendamment des autres. L'unité de ce retable, évidente quand on en considère l'ensemble, tient à l'esprit qui, audelà de la diversité des thèmes, est essentiellement le même dans tous les volets de l'œuvre (Domingo $1961: 7-8$ ).

D’ailleurs le préfacier ne parle pas de nouvelles, mais de romans : « La permanence du décor, d'un roman à l'autre, la référence constante au buriti et à d'autres totems [...] ne suffiraient pas, par elles-mêmes, à structurer organiquement une œuvre aussi vaste si tous les panneaux du retable n'étaient habités par le même démon-écrivain. " Voilà une autre forme d'incantation qui pourrait consoler un éditeur dont l'ambition était de faire connaître toute l'œuvre de Guimarães Rosa.

Curieusement, les commentaires qui s'inspirent ou dérivent des pistes fournies par cette préface vont parfois surlire la sensualité sous-tendant l'envoûtement du texte. Si Le Canard enchaîné salue la réhabilitation d'un « érotisme de bon aloi », Le Courrier de Vervière ne conseille la lecture de « Dão-lalalão » « qu'aux adultes » (Dubois 1961) ! La Feuille d'Avis de Neufchâtel construit plus solidement l'argument pour, au bout du compte, rendre compatible l'union des corps et l'union des âmes :

Buriti, du romancier brésilien João Guimarães Rosa, se situe sous le signe de l'impressionisme panique. D'emblée on est plongé dans ce mystère de forêt vierge, où végétaux, animaux, humains, et la terre elle-même semblent ne connaître qu'une loi, celle du désir le plus élémentaire, et le plus absolu. Un seul but réalisé dans une extase permanente : l'union, la communion charnelle (Anonyme 1961).

Virgile et la référence au dieu Pan vont aussi connaître quelques avatars. Dans La Tribune de Genève, James Ramoni se contente de mentionner le lyrisme du poète de Mantoue : "Mille touches grisantes, signature du premier style de Virgile, selon M. Jean Bayet, doivent jeter l'âme dans un état permanent d'ivresse lyrique. De même, l'auteur de "Buriti" use davantage de l'incantation que du récit pour subjuguer son lecteur. » (Ramoni 1962). Mais c’est pour conclure par

20 En fait Corpo de baile se divise en sept longues nouvelles, d'abord éditées en deux tomes, puis redivisées en trois parties presque autonomes. 
la foisonnante exubérance du Brésilien : « Nous voyons bien que, dans cette œuvre inégale, le grand Pan, contrairement à une prédiction célèbre, n'est pas mort. On s'aperçoit toutefois que le Pan brésilien est mille fois plus exubérant que son collègue romain : que de singes, que de cacatoès, que d'urubus ! „ Guy Dumur (1961) va plus loin, en interposant « irrésistiblement » entre Virgile et Guimarães Rosa une lecture écran qui n’est pas dans la préface de Xavier Domingo : celle des premiers romans de Jean Giono et de son cycle panique, avec en arrière-plan le retour d'une écriture possiblement régionaliste, du terroir. La trilogie, Colline, Un de Baumugnes, Regain, est citée par une presse syndicale belge (J. B. 1961) ; de façon plus attendue dans la presse du Midi, un journaliste de Nîmes mentionne la pastorale du Serpent d'Étoiles :

La terre, le sertão, n'est plus un objet, elle accède ici à sa vraie grandeur : elle redevient la terre nourricière, la terre qui nourrit et enrichit l'âme de ceux qui la parcourent, elle est un être vivant, elle est l'Autre sans qui aucun amour n'est possible (Bonniès 1961).

Un mot manquait encore s'agissant de littérature latino-américaine : le baroque. C'est Yves Berger qui l'ajoute dans les colonnes de L'Express : dans cette « grande fête de la nature sauvage, musique mystique et délirante que rythme une imagination baroque », tout est " démesure » et " excès », et Guimarães Rosa est un « Giono multiplié par dix » (Berger 1961). Il n'est donc pas surprenant que cette hybris à la lisière du fantastique ait intéressé Planète, la revue bimestrielle née précisément en 1961, à l'initiative de Jacques Bergier et Louis Pauwels pour lesquels « Rien de ce qui est étrange ne nous est étranger ! » : y paraît en 1962 une nouvelle extraite de Primeiras estórias (1962), " La troisième rive du fleuve », traduite par Maria Frias et Maurice Pons.

La nature " envoûtante » et la sensualité frappent peut-être encore plus fortement la critique lors de la publication, en 1962, de Les Nuits du sertão :

Dans un cadre naturel singulièrement envoûtant, la sensualité jette Lalinha dans les bras de iô Liodoro et Gloria dans ceux du rustre et lubrique Gualberto Gaspar, mais Gloria sera sauvée par l'amour de Miguel, qui après un long éloignement la rejoint. Tableau de mœurs dans une région aux singuliers prestiges, les forces de l'amour et de la nature s'y conjuguent pour élever les notes d'un chant tantôt pur et tantôt sensuel, puissant comme le grand palmier royal qui se dresse tel un symbole impérissable sur les terres du sertão (Anonyme 1963).

Dans Avenir Luxembourg, J. Amica semble répondre à Yves Berger : prétendre que Guimarães Rosa est un Giono décuplé est excessif et inexact. D’une part, Giono « est "notre” Giono », de l'autre, " l'écrivain brésilien est plus dense que Giono, plus essentiel en quelque sorte » (Amica 1962). La confrontation est retravaillée par le critique belge Jacques Parisse : chez Guimarães Rosa, l'intégra- 
tion entre l'homme et la nature est totale, au contraire de Giono ou d'Henri Bosco, et débouche sur le dépaysement exotique (inversant au passage la leçon de Xavier Domingo).

En lisant « Les Nuits du Sertão » on se souvient de certaines pages de " Batailles dans la Montagne » de l'écrivain de Manosque et de la longue et admirable dissertation sur le vent qui faisait agir les personnages de « Mallicroix » d'Henri Bosco. Mais chez Giono, mais chez Bosco, l'homme ne s'intégrait pas entièrement à la nature qui ne restait, en fin de compte, que le ferment parfois artificiel et littéraire de leurs actions. João Guimarães Rosa, le plus important des écrivains brésiliens, n'a guère dissocié la nature luxuriante des grandes savanes et des épaisses forêts et les hommes totalement conditionnés par les éléments. On peut aller jusqu'à dire que dans cette œuvre profonde où le souffle se perd, Rosa, démiurge tout puissant et inspiré, accouple avec une imagination et un sens descriptif quasi satanique, héros du récit et décors. L'exotisme, le dépaysement - qui pourrait bien être la qualité fondamentale du vrai roman - sont poussés ici jusqu'à leur paroxysme. (Parisse 1962).

Marcel Brion reprend la plume et les pistes gnostiques de Xavier Domingo :

Ce livre n'exige pas une interprétation littéralement ésotérique, mais plutôt une connaissance de ce que veut ce réalisme panique exprimé et des articulations principales de ce « langage de contemplation » employé par Guimarães Rosa. Il suffit d'avertir le lecteur que les choses, dans ce livre, ne sont pas seulement ce qu'elles paraissent être - et sont effectivement - au premier coup d'œil, et qu'elles se prolongent sur un autre plan de la réalité, vers laquelle nous conduisent les allusions à Ruysbroeck, les rappels de Plotin. L'entreprise n'est pas toujours aisée, mais elle mérite d'être tentée (Brion 1962).

Mais il va au-delà en ouvrant la voie à de futures lectures (Utéza 1994) mettant en relation l'œuvre de Guimarães Rosa et les philosophies orientales : «Celuilà seul qui sait apercevoir les deux plans du réel et les embrasser simultanément saisit l'équivalence de l'objet et de son ombre, et la leçon de ce sentier tracé à travers la forêt, qui est le Chemin : ce que les Chinois appellent le Tao. » (Brion 1962)

Enfin, la langue - le « langage de la contemplation » disait la préface de Xavier Domingo qui signalait aussi l'usage « abondamment et avec bonheur [de] l'argot sertanejo »-, est un autre axe de commentaire, auquel était déjà sensible Le Nouveau Journal de Montréal à propos de Buriti :

Le ton est poétique, et la beauté de la langue qui s'identifie étroitement aux choses, en efface le régionalisme ; elle devient palpable et visible. Seul, " Dao Lalalao " est un roman ; « Le message du Morne », et « La fête de Manuelzão », qui complètent « Buriti », sont des chants (Anonyme 1961a).

Et que Le Progrès ressent à nouveau avec Les Nuits du sertão : 
Mais ce second livre [...] est peut-être plus fascinant encore, car y est encore plus habile l'étrange incantation des phrases enfilées et comme vissées les unes aux autres, n'existant que les unes par les autres, comme d'inséparables points de tapisserie dont aucun n'a de signification hors de l'ensemble. Phrases de tonalité d'une monotonie parfois prolongée jusqu'à la tension, puis soudain coupées des stridences exaspérées (Anonyme 1961).

Cette langue si particulière amène les critiques à porter aussi leur attention sur la traduction et l'effort de recréation qu'elle exige. Et les éloges ne manquent pas : « admirable » (Dedet 1961), « particulièrement heureuse » (Berthier 1961), « éblouissante » (Franklin 1962). Dans Les Lettres françaises, on note à propos des Nuits du sertão « que peu d'auteurs étrangers ont bénéficié d'une traduction comparable, semble-t-il, à celle de J.-J. Villard » (Juin 1963). Après avoir souligné qu' " on ne raconte pas, on ne juge pas Les Nuits du Sertão », on écrit dans Les Livres qu'il faut « se laisser envoûter à son tour par la magie de João Guimarães Rosa, heureusement servie par la version de J. J. Villard » (R.C. 1963). Le Progrès souligne aussi que « l'envoûtement » qui résulte de la lecture de Buriti et des Nuits du sertão doit " beaucoup, sans doute, grâce à son traducteur, J.-Jacques Villard " (B. G. 1962). Il est probable que ces jugements ne sont pas fondés sur une connaissance de l'original, mais sur l'intuition que communique le texte français et de ce qu'il laisse entendre de l'écriture si nouvelle de Guimarães Rosa. On relève d'ailleurs l'adverbe prudent du Canard enchaîné, dans l'article consacré à Buriti : « la traduction de M. J. J. Villard est sûrement toute bonne puisqu'elle a laissé à l'œuvre toute sa fraîcheur, toute sa virile spontanéité ».

En somme, la réception critique des deux premiers volumes français de Corpo de baile, significativement abondante, est globalement positive, et salue " le talent irrécusable d'un grand écrivain », et implicitement l'habilité de son traducteur. On ne dispose pas d'un dossier de presse si fourni pour l'édition de Diadorim chez Albin Michel, en 1965. On ne peut que remarquer le choix discutable du titre, qui s'impose (avec l'aval de l'auteur) comme un effacement de la complexité de Grande sertão : veredas, et comme un déplacement fâcheux de la clé de voûte du roman, à comparer avec d'autres solutions. L'éditeur étasunien Knopf a opté pour The Devil to Pay in the Backlands (1963, trad. Harriet de Onis et James L. Taylor) ; Curt Meyer-Clason a conservé Grande Sertão dans l'édition allemande (1964), comme d'ailleurs Edoardo Bizarri et l'éditeur italien Feltrinelli (1970) ; Seix Barral et Ángel Crespo ont transposé en espagnol : Gran Sertón : veredas (1967). En France, un troisième volet, Hautes Plaines, est publié par Seuil en 1969, mais ni le cœur ni l'esprit n’y sont plus : João Guimarães Rosa a été emporté le 19 novembre 1967. La destinée d'une œuvre et de sa réception tient à de multiples facteurs, une série d'agents, la centralité ou non de la langue, la complexité et la nouveauté de l'écriture, les structures de réception, et aussi ces accidents qui laissent le texte orphelin, changent le rapport aux droits d'auteur. Sans 
compter que l'envol est fauché dans son élan : à la veille de sa mort, Guimarães Rosa semblait un solide prétendant au Prix Nobel, et Roger Caillois aurait même affirmé que le jury de l'académie suédoise avait déjà convenu qu'il serait le lauréat de 1968 (Anonyme 1965).

\section{Après 1980}

C'est ainsi qu'à l'effervescence des années 1960 succède le silence de plus d'une décennie. Le Seuil a publié ce qui avait été programmé, en renonçant à la sortie des Premières Histoires, pourtant déjà traduites par Villard. C'est justement ce livre de nouvelles qui relance, en 1982, la réception, de façon à nouveau éphémère puisqu'il faut attendre encore près de dix ans pour que la publication de Métailié connaisse une suite. À titre de comparaison, dès 1970, le public allemand a déjà accès à presque toute l'œuvre, à l'exception de Tutaméia et de Sagarana.

On peut s'étonner de ce décalage et de la frilosité française qu'explique peut-être une fois encore la difficulté de la langue car, comme nous l'avons déjà signalé, les années quatre-vingt voient se combler progressivement le déficit de traductions de la littérature brésilienne. Il est sans doute établi que Guimarães Rosa est un des très grands écrivains de la littérature mondiale, comme l'écrivent Jorge Coli et Antoine Seel en 1982, à la sortie de Premières histoires :

Joyce perdu dans une province du Brésil. Homère chantant le sertão du Minas Gerais : Guimarães Rosa a déjà été l'objet de comparaisons les plus flatteuses. Plus encore, qu'un des grands maîtres de la littérature latino-américaine, l'on reconnaît en lui un des meilleurs écrivains de notre époque. (Coli/Seel 1982).

Mais cette grandeur impressionne et dissuade. Dans les années quatre-vingtdix, Jacques Thiériot convainc partiellement Le Seuil de reprendre l'entreprise interrompue en 1969, et Albin Michel juge également le moment opportun pour remettre l'ouvrage sur le métier. En 1991, Maryvonne Lapouge-Pettorelli signe pour cette maison-ci une nouvelle traduction de Grande sertão : veredas, toujours sous le titre Diadorim, avec une préface de Mario Vargas Llosa ; version qui va aussi connaître une édition de poche en 10/18 (1995). Et Jacques Thiériot livre successivement Toutaméia. Troisièmes histoires en 1994 (Seuil), et Sagarana en 1997 (Albin Michel). Dans la foulée, il traduit "Meu tio o iauaretê » qu'Albin Michel publie en 1998 sous le titre Mon oncle le jaguar, avec une jaquette reproduisant un détail de Forêt vierge au soleil couchant, d'Henri Rousseau. Le primitif se stylise. En reprenant le titre en 2000, la collection de poche 10/18 adopte une stratégie visuelle proche de son édition de Diadorim : une 
photo de vacher nordestin, tout de cuir vêtu comme il se doit, avançant à cheval dans un paysage d'épineux. La légende de la reprise de 1995 précisait en revanche : «Gaucho brésilien de la Campanha »! L’art de dépayser le dépaysement.

Pour être complet, en excédant la coupure de 2000, précisons que Mathieu Dosse retraduit la nouvelle extraite de Estas estórias, et le reste du recueil paru initialement en 1969 (de façon posthume donc), même si plusieurs des textes avaient été publiés avant 1967. L'édition Chandeigne se raccroche au titre déjà connu du public : Mon oncle le jaguar \& autres histoires (2016). La même année, la maison édite une version de la première nouvelle de Sagarana, Sept-de-Carreau. L'âne du sertão, avec des illustrations d'Olivier Besson qui est à l'origine de ce projet.

Quant à l'accueil critique des années quatre-vingt-dix, il change sensiblement de celui des années soixante. Non seulement le contexte politique a changé, la littérature brésilienne a gagné une place plus autonome, mais le lecteur n'est plus sous l'impact de la nouveauté. Il lit un classique. Quoique ... Guimarães Rosa a sa place aux côtés des plus grands, mais Pierre Lepape, critique littéraire du Monde, s'étonne à propos de Diadorim que, « l'un des plus grands livres de ce siècle [...] ne soit pas aussi connu que l'Ulysse de Joyce ou que Les Cantos d'Ezra Pound » (Lepape 1998). Dans le même journal, Yves-Marie Labe se demande « comment rendre compte des 500 pages de Diadorim, ce livre-phare de la littérature brésilienne écrit avec une densité hors du commun par un écrivain de la taille de Jorge Luis Borges ou Thomas Mann ? „(Labe 1995). Pour Alain Bosquet qui commente alors Toutaméia, Guimarães Rosa, bien qu' à la fois le James Joyce et le Jorge Luis Borges des lettres brésiliennes », « est bien plus que cela : un écrivain diaboliquement saisi par le démon d'une langue qu'il veut sans cesse renouveler, maltraiter, refaire, enrichir sans limites » (Bosquet 1994). Aux yeux de Claudio Magris et de Pierre Lepape, à propos de Diadorim, le régionalisme reste un piège, l'exotisme et la sociologie le deviennent :

Avec pour décor le plateau brésilien, l'expérience errante des jagunços, les cavaliers et bouviers de ces solitudes, Grande sertão fait de ce monde, sans aucun exotisme, un paysage universel de la vie, de son nuage de poussière et de sa signification. C'est une odyssée qui repropose sous une forme nouvelle et originale les interrogations fondamentales de l'expérience humaine, la question de savoir si Ulysse rentrera à la maison avec une identité confirmée ou condamné à être définitivement Personne (Magris 1992).

Pendant les cinq cents pages de Diadorim, nous n'allons pas découvrir en touristes une terre étrange, parcourue par de non moins étranges aventuriers, les jagunços, nous allons errer avec eux, au milieu d'eux, selon les lois propres de cet univers, sa logique, sa démesure, sa folie, sa sagesse, sa temporalité, son espace, son langage. L'universalité du roman de Guimarães Rosa tient à la réussite de cette gageure : inscrire le Sertão comme 
une réalité commune à tous ses lecteurs : à aucun moment comme un spectacle exotique. (Lepape 1991).

Les jugements sur Sagarana ne sont pas différents :

Surtout Guimarães Rosa transcende sa documentation régionale, comme Giono ou Ne pas couper de préférence inventant leur sud. Il est du Minas comme Joyce de Dublin (Anonyme 1997).

Universel et régionaliste, profondément universel parce qu'intensément régionaliste, Guimarães Rosa déniche l'homme, tout l'homme au fond de sa province ingrate (Soublin 1997).

Le regard aussi a changé. Dans les années 1960, Marcel Brion, responsable de la diffusion de la littérature luso-brésilienne aux Nouvelles littéraires, était l'un des rares à être un peu familier du domaine (Cf. Rivas 1995 : 278). Depuis, le monde des "spécialistes " s'est modestement étoffé, le maillage des passeurs s'est légèrement resserré. Dans les années quatre-vingt, Clélia Pisa et la traductrice Alice Raillard ont des fonctions de conseil, pour ne pas dire d'agent, dans quelques maisons d'édition ; et les traducteurs prennent des initiatives. Le Brésilien Jorge Coli et Antoine Seel, qui ont converti en français Euclides da Cunha et Graciliano Ramos, écrivent dans la presse spécialisée. Le journaliste et écrivain Jean Soublin connaît bien le Brésil. L'universitaire Francis Utéza déchiffre Toutaméia lors de sa sortie (Utéza $1997: 19-21)^{21}$, le comparatiste Pierre Rivas écrit dans La Quinzaine littéraire à propos de Sagarana:

On distingue généralement deux pôles dans la littérature brésilienne : le cosmopolitisme ouvert sur la modernité internationale, littérature des grandes métropoles atlantiques, et l'enracinement dans l'intérieur, le sertão, la brousse, tradition régionaliste et rurale. Antagonisme propre « aux deux Brésils » entre modernité et tradition. L'œuvre de João Guimarães Rosa parvient à transcender cette opposition et fonde ainsi, dans la tradition de Faulkner, tenu lui aussi longtemps comme écrivain régionaliste, cette littérature de transculturation, où les techniques de la modernité viennent féconder une matière rurale, longtemps réduite au pittoresque et au folklore (Rivas 1997 : 5).

Leurs analyses se font ainsi non seulement à partir des traductions, mais à partir des textes originaux et des études qui y ont été consacrées. Auteur d'une somme de référence sur les références cryptées de l'écrivain du Minas, et conseiller de Jacques Thiériot pour la traduction de Toutaméia, Francis Utéza éclaire la dimension alchimique et métaphysique du recueil, fondue dans le corps du langage commun :

21 Voir aussi la recension de Michel Riaudel : «Sagarana. João Guimarães Rosa ». Infos Brésil, $\mathrm{n}^{\circ}$ 125, Ivry-sur-Seine, 15 mai 1997. 
Ce décodage doit aussi prendre garde au langage pseudo tous les jours : indiquée peut aussi signifier marquée par la Diké, et - problème pour le traducteurs - dans le bois brésilien - madeira - se cache aussi la, si l'on peut dire, pure et simple matière, étymologiquement entendue - matéria (Utéza 1994 : 21).

L'explication savante ne retire rien aux déclarations d'envoûtement. En une allusion à l'entretien accordé par Guimarães Rosa à Gunter Lorenz, Frédéric Pagès évoque le « mage du verbe ", le « forgeron du parler maternel » (Pagès 1997). Dans un registre similaire, Alice Raillard parle de son " langage magique " (Raillard 1982), Jacques Fressard de " la magie d'un langage d'une richesse et d'une beauté peu communes " (Fressard 1991), Pierre Lepape, de «l'éblouissant roman de geste [Diadorim] » (Lepape 1991) et de « l'envoûtement garanti » (Lepape 1998) par la lecture de Mon oncle, le jaguar. Enfin Jean Soublin note la « transe » qui suscite chez le lecteur ce langage « riche, complexe, difficile » (Soublin 1997).

\section{La tâche du traducteur}

En guise de bilan et d'échappée, nous voudrions pour conclure insister sur la création stylistique et langagière de cette œuvre qui fait une grande part de sa richesse, mais constitue aussi un défi, sinon un obstacle, à sa traduction et sa réception. Soucieux de la diffusion de son œuvre, João Guimarães Rosa n'a pas ménagé ses efforts de son vivant pour répondre aux doutes de ses traducteurs. La thèse de Nícia Adan Bonatti a recensé 372 lettres. Toutes deux déjà publiées, la correspondance avec Curt Meyer-Clason s'est étalée du 23 janvier 1958 au 27 août 1967, celle avec Edoardo Bizarri, du 18 octobre 1959 au 27 août 1967. En attente de publication, celle avec Harriet de Onís couvre la période du 19 novembre 1958 au 25 octobre 1966 ; l'écrivain échange avec Jean-Jacques Villard du 7 juillet 1961 au 25 avril 1967 ; la plus courte est celle avec Ángel Crespo, qui s'étend du 26 février 1964 au 21 février 1967. Mais le rapport qu'il établit avec ses interlocuteurs éclaire quelque chose du lien qu'il cherche à instaurer avec son lecteur de manière générale : une logique initiatique, quelque chose du sage qui révèle beaucoup mais ne livre pas tout. Il accompagne sur la voie de vérités plus profondes.

La réception d'une œuvre littéraire n'est pas une étape ultérieure, extérieure à la création, elle en est au contraire une dimension intrinsèque. Chaque lecture la fait advenir et en complète provisoirement la signification. Dans le cas de Guimarães Rosa, ce constat se manifeste notamment par l'envie que ses 
textes suscitent d'être traduits et sans cesse retraduits. Mieux, la traduction est, à ses yeux comme pour nous, constitutive de son art :

Moi, quand j'écris un livre, je fais comme si j'étais en train de « traduire », à partir de quelque très ancien original, existant ailleurs, dans le monde astral ou le « plan des idées », des archétypes, par exemple. Je ne sais jamais si je la réussis ou si je la rate, cette " traduction ». De sorte que, lorsqu'on me (re)-traduit en un autre idiome, je ne sais pas davantage, en cas de divergence, si ce n'est pas le Traducteur qui, de fait, a trouvé la solution et rétablit la vérité de l'« original idéal ", que j’avais dénaturée (Lettre à Edoardo Bizzarri 4 septembre 1963. In Rosa 1981 : 63-64).

Peut-être y a-t-il là matière à se consoler de cette réception accidentée, inachevée ... - parce qu'en fin de compte sans cesse à recommencer.

\section{Bibliographie}

Aguiar, Márcia V. M. de (2017) : «A publicação de Guimarães Rosa na França dos anos 1960 ». In Reimão, Sandra/Riaudel, Michel (eds.) : Livros, literatura e história: passagens BrasilFrança. Florianópolis : Escritório do Livro, pp. 79-102.

Aguiar, Márcia/Riaudel, Michel (2018) : « João Guimarães Rosa: un auteur à remémorer pour le commémorer ». In : Herrera, Raquel Molina/Castaño, Victoria Ríos (eds.) : Colloquia. Paris : Sorbonne Université. <https://colloquiasal.files.wordpress.com/2019/03/colloquia6f.pdf>. Azevedo, Aluísio (1961) : Le Mulâtre. Trad. Pierre-Manoel Gahisto. Paris : Plon.

Bonatti, Nícia Adan (1999) : Entre o amor da língua e o desejo : a tarefa sem fim do tradutor. Campinas : IEL/Unicamp. <http://repositorio.unicamp.br/bitstream/REPOSIP/271083/1/ Bonatti_NiciaAdan_D.pdf>.

Coubertin, Pierre (2015) : Olimpismo. Recueil de textes. Ed. Müller, Norbert/Nelson Schneider. Trad. Luiz Carlos Bombassaro. Porto Alegre : EdipucRS.

Domingo, Xavier (1961) : « Préface ». In : Rosa, João Guimarães : Buriti. Trad. Jean-Jacques Villard. Paris : Seuil, pp. 7-11.

- (13 février 1959) : Rapport de lecture de Corpo de baile. Fonds Seuil/IMEC.

Flamand, Paul (1979) : Sur le Seuil. Paris : Seuil.

José Guilherme Klingelhoefer. In : Portugal, Dicionário histórico. <https://www.arqnet.pt/ dicionario/klingelhoefer.html>.

Lacouture, Jean (2010) : Paul Flamand, éditeur. Paris : Éditions des Arènes.

Liscano, Juan : "Avant-propos » (1958). In : Les vingt meilleures nouvelles de l'Amérique Latine. Paris : Seghers, pp. 11-18.

Mollier, Jean-Yves (1995) : « Paris, capitale éditoriale des mondes étrangers ». In : Marès, Antoine/Milza, Pierre (eds.) : Le Paris des étrangers depuis 1945. Paris : Publications de la Sorbonne, pp. 373-394.

- (2012) : «Un essai de dépassement des communautés partisanes : la collection "Méditerranée" au Seuil pendant la guerre d’Algérie (1945-1962) ». In : Cahiers de la Méditerranée moderne et contemporaine 85, Nice/Centre de la Méditerranée Moderne et Contemporaine, pp. 49-57. 
Moraes, Vinícius de (1953) : Cinq élégies. Trad. Jean-Georges Rueff. Paris : Seghers, coll. «Autour du monde».

Pailleron, Marie-Louise (1919) : François Buloz et ses amis. La vie littéraire sous Louis Philippe. Paris : Calman-Lévy.

Riaudel, Michel (2017) : «Teria havido um boom latino-americano ». Trad. Márcia Aguiar. In : Reimão, Sandra/Riaudel, Michel (eds.) : Livros, literatura \& história: Passagens Brasil-França. Florianópolis : Escritório do livro, pp. 11-47.

Rivas, Pierre (1995) : Encontro entre literaturas. Tradução Durval Artico/Maria Letícia Guedes Alcoforado. São Paulo : Hucitec.

Rosa, João Guimarães (1961) : Buriti. Trad. Jean-Jacques Villard. Paris: Seuil.

- (1981) : Correspondência com seu tradutor italiano, Edoardo Bizzarri. São Paulo : Queiroz; Instituto Cultural Ítalo-Brasileiro, 1981.

- (1962) : Les nuits du sertão. Trad. Jean-Jacques Villard. Paris : Seuil.

- (1965) : Diadorim. Trad. Jean-Jacques Villard. Paris : Albin Michel.

- (1969) : Noites do sertão. Rio de Janeiro : José Olympio ( $4^{\mathrm{e}}$ édition).

- (1969) : Hautes plaines. Trad. Jean-Jacques Villard. Paris : Seuil.

- (1982) : Premières Histoires. Trad. Inès Oseki-Depré. Paris : Métailié.

- (1991) : Diadorim. Trad. Maryvonne Lapouge-Petorelli. Paris : Albin Michel.

- (1994) : Toutaméia. Troisièmes histoires. Trad. Jacques Thiériot. Paris : Seuil.

- (1994) : Ficção completa em dois volumes. Rio de Janeiro : Nova Aguilar.

- (1997) : Sagarana. Trad. Jacques Thiériot. Paris : Albin Michel.

- (1998) : Mon oncle le jaguar. Trad. Jacques Thiériot. Paris : Albin Michel.

- (2016) : Mon oncle le jaguar \& autres histoires. Trad. Mathieu Dosse. Paris : Chandeigne.

- (2016) : Sept-de-Carreau, l'âne du sertão. Trad. Michel Riaudel, illustrations d'Olivier Besson. Paris : Chandeigne.

Serry, Hervé (2002) : « Constituer un catalogue littéraire. La place des traductions dans l'histoire des Éditions du Seuil ». In : Actes de la recherche en sciences sociales 144, pp. 70-79.

- (2008) : Les Éditions du Seuil, 70 ans d'histoires. Paris : Seuil/IMEC.

Veríssimo, Érico (1955) : Le Temps et le Vent. Le Continent. Trad. Jean-Jacques Villard (portugais). Paris : René Julliard.

Villard, Jean-Jacques (2008) : Nino Bixio, uomo, marinaio, patriota. La vita avventurosa dell'eroe garibaldino nel racconto del pronipote. Milan : Viennepierre Edizione.

Villard, Théodore (1900) : Les fleurs à travers les âges et à la fin du XIX siècle. Paris : Armand Magnier.

\section{Correspondances et lettres}

Bastide, Roger (1959) : Lettre à Michel Chodkiewicz, 20 janvier. Fonds Seuil - IMEC.

Chodkiewicz, Michel (1959a) : Lettre à Antonio Bandeira, 29 mai 1959. Fonds Seuil - IMEC.

- (1959b) : Lettre à Olga Obry, 30 décembre 1959. Fonds Seuil - IMEC.

- (1959c) : Lettre à Roger Bastide, 16 janvier 1959. Fonds Seuil. IMEC.

- (1960): Lettre à Everaldo Dayrell de Lima, 7 janvier 1960. Fonds Seuil - IMEC.

Denis, Pierre (1959) : Lettre à Michel Chodkiewicz, 8 octobre 1959. Fonds Seuil - IMEC.

Éditions Albin Michel (1958): Lettre à José Olympio Editores, 23 mars 1958. Fonds João

Guimarães Rosa - IEB - USP. 
Rosa, João Guimarães : Correspondance avec Michel Chodkiewicz. Fonds Seuil. IMEC.

-: Correspondance avec Pierre Seghers. Fonds João Guimarães Rosa - IEB - USP.

-: Correspondance avec son traducteur français, Jean-Jacques Villard. Fonds João Guimarães Rosa - IEB - USP [1961-1967].

Seghers, Pierre (1957) : Lettre à Antonio Tavares Bastos, 25 juin. Fonds João Guimarães Rosa - IEB - USP.

Villard, Jean-Jacques (1959) : Lettre à Michel Chodkiewicz, 25 novembre. Fonds Seuil - IMEC.

\section{Articles de presse}

Alvim, Gilda Césario (1961) : "Guimarães Rosa em francês ». In : Jornal do Brasil. Rio de Janeiro, 22 août 1961. Billet de Paris. Fonds João Guimarães Rosa - IEB - USP.

Amica, J. (1962) : « L'épopée primitive dans un paradis retrouvé. Les nuits du sertao ». In : Avenir Luxembourg, 2 octobre. Fonds João Guimarães Rosa - IEB - USP.

Anonyme (1961): « Buriti ». In : Feuille d'Avis, Neufchâtel, 23 juin. Fonds João Guimarães Rosa - IEB - USP.

Anonyme (1961): «Le Nouveau Journal a lu pour vous ». In : Le Nouveau Journal, Montréal, 30 septembre (a). Fonds João Guimarães Rosa - IEB - USP.

Anonyme (1963) : « Les nuits du sertão ». In : Bulletin Critique Livre Français, mai. Fonds João Guimarães Rosa - IEB - USP.

Anonyme (1965): «Guimarães Rosa candidato ao Prêmio Nobel na Itália ». In : O Globo, Rio de Janeiro, 3 juin. Fonds JGR - IEB-USP.

Anonyme (1997) : [sans titre]. In : Provençal/Méridional, 4 mars.

Berger, Yves (1961) : «À La gloire des animaux, des fous et des montagnards du Brésil ». In : L'Express, Paris, 27 avril. Fonds João Guimarães Rosa - IEB - USP.

Berthier, Pierre (1961) : « Du reportage au roman ». In : La Cité, Bruxelles, 20 avril. Fonds João Guimarães Rosa - IEB - USP.

Bonniès, Henri (1961) : «Buriti ». In : Nîmes Soir, 20 avril. Fonds João Guimarães Rosa IEB - USP.

Bosquet, Alain (1994) : "Le fou des mots ». In : Magazine littéraire, Paris, avril.

Brion, Marcel (1962) : [sans titre]. In : Les Nouvelles littéraires, Paris, 11 janvier. Littérature sud-américaine. Fonds João Guimarães Rosa - IEB - USP.

Coli, Jorge/Seel, Antoine (1982) : «Le Brésil de Guimarães Rosa ». In : Le Monde, Paris, 17 décembre.

Dedet, Christian (1961) : « Sud profond. João Guimarães Rosa : Buriti ». In : Arts, 13 septembre. Fonds João Guimarães Rosa - IEB - USP.

Dubois, Louis (1961) : « Une voix du Brésil, Buriti ». In : Le Courrier, Vervière, 19 mai. Fonds João Guimarães Rosa - IEB - USP.

Dumur, Guy (1961) : «Buriti: un monde de chair et de rêves ». In : France Observateur, 4 mai. Fonds João Guimarães Rosa - IEB - USP.

Fressard, Jacques (1991) : «Guimarães Rosa, le magicien ». In : La Quinzaine littéraire 572, 16 février.

J. B. (1961): « Buriti par João Guimarães Rosa ». In : Syndicats, Bruxelles, 3 juin. Fonds João Guimarães Rosa - IEB - USP.

Labe, Yves-Marie (1995) : « Le roman universel du Sertao ». In : Le Monde, Paris, 20 mai. 
Lefèvre, René (1961) : « Buriti ». In : Le Canard Enchaîné, 3 mars. Fonds João Guimarães

Rosa - IEB - USP.

Lepape, Pierre (1991) : "L'épopée de João Guimarães Rosa ». In : Le Monde, Paris, 8 février.

- (1998) : "La nuit de la métamorphose ». In : Le Monde, Paris, 20 mars.

Magris, Claudio (1992) : "Le jardin secret de Claudio Magris: Grande Sertão: Veredas ».

In : Quinzaine littéraire, Paris, 1 août. Numéro spécial: Le jardin secret de 36 écrivains.

Pagès, Frédéric (1997) : « João Guimarães Rosa. Sagarana ». In : Les Inrockuptibles, 19 février.

Parisse, Jacques (1962) : « Les nuits du sertão par João Guimarães Rosa ». In : Combat, Liège,

17 novembre. Fonds João Guimarães Rosa - IEB - USP.

Raillard, Alice (1982) : « Les héros du Sertan ». In : La Croix, 6 novembre.

Ramoni, James (1962) : « Buriti: trois romans virgiliens ». In : Tribune de Genève, Genève,

13-14 mai. Fonds João Guimarães Rosa - IEB - USP.

Riaudel, Michel (1997) : « Sagarana. João Guimarães Rosa ». In : Infos Brésil 125, Ivry-surSeine, 15 mai.

Rivas, Pierre (1997) : « João Guimarães Rosa. Sagarana ». In : La Quinzaine littéraire, 16 février.

Sion, George (1961) : «Parmi tant de fureurs ... ». In : Phare, 18 juin 1961. Fonds João Guimarães Rosa - IEB - USP.

Soublin, Jean (1997) : « Fulgurances et musique d'une langue locale ». In : Le Monde, Paris, 17 janvier.

Ténèze, Roger (1961) : « Buriti ». In : Voix du Nord, Lille, 27 octobre. Fonds João Guimarães Rosa - IEB - USP.

Utéza, Francis (1994) : « L’ésoterisme de “Toutaméia” ». In : Infos Brésil 93, Ivry-sur-Seine, juin. 
